ity, it is because they examine one lode of thought in great depth. The exercise proves valuable partly because its extremely clear focus shows how much has been perforce omitted and how much we still have to learn.

\section{Functional significance of human female orgasm still hypothetical}

\section{Nicholas Pound and Martin Daly \\ Department of Psychology, McMaster University, Hamilton, Ontario, Canada, L8S 4K1 \{daly; pound\}@mcmaster.ca}

Abstract: Human males are more polygamously inclined than females However, there is substantial within-sex variation in polygamous inclinations and practices. This is acknowledged by Gangestad \& Simpson but we pose the question: Is the target article's “strategic pluralism” pluralistic enough? In addition, we argue that the hypothesis that the female orgasm is an adaptation for post-copulatory female choice between rival ejaculates demands more research.

It has long been evident that in people, as in most other mammals, males are more polygamously inclined than females, while the latter are choosier in evaluating possible copulatory partners. These sex differences are readily understood in the light of the BatemanWilliams-Trivers theory of sexual selection and parental investment (e.g., Daly \& Wilson 1978; Symons 1979).

Many people, including some evolutionists, dislike these truisms, but no one has mustered an evidence-based case against them. Nor are they called into question by Gangestad \& Simpson (G\&S), who are concerned with subtler issues: Why are these sex differences true only on average, with much overlap between women and men, and why is there so much variation in polygamous inclinations and practices within each sex? The target article reviews some relevant hypotheses and recent empirical evidence, including the authors' own contributions, and it should stimulate additional discoveries. We wish to draw attention to two issues in need of further research.

First, we wish to pose the question: Is the target article's "strategic pluralism" pluralistic enough? The authors have demonstrated large individual differences in "sociosexuality," a dimension which they characterize here as being primarily a matter of variable willingness "to have sex without commitment" and "to engage in short-term mating” (sects. 4.5.3 and 5.4). A central hypothesis is that "restricted" women who demand commitment from longterm partners are opting for paternal investment in a biparental partnership, whereas "unrestricted" willingness to forego such commitment entails trading off the material benefits that an investing partner would provide, in exchange for a "good genes" payoff. Without denying the plausibility of such trade-offs, we would suggest that this scheme is not pluralistic enough, especially with respect to relatively "unrestricted" female strategies. Cuckolding a long-term partner in order to recruit better genes through adultery is surely quite different from the polyandrous strategies of women who consider men too unreliable to bother seeking commitment. To suggest that an unrestricted female strategy of shortterm mating entails giving up material compensation is curiously forgetful of the world's "oldest profession": the trade-off for women choosing whether to adopt a short-term mating strategy is in large measure a trade-off between material compensation now versus (promised) material compensation in the future, and a big determinant must be one's assessment of the trustworthiness of promises. Moreover, insofar as good genes sexual selection has been an important force in the evolution of the female psyche, we might expect that women would adjust "prices" for both shortterm and long-term mates as a function of genetic quality cues.

The second issue demanding more research concerns the hypothesis that the female orgasm is an adaptation for post-copulatory female choice between rival ejaculates, and hence between potential sires (Baker \& Bellis 1995). G\&S invoke this intuitively appealing notion in the context of arguing that women bias the chance of conception towards more symmetrical partners (sect. 4.5.1), but although their evidence that men's fluctuating asymmetry (FA) is negatively correlated with their sex appeal is persuasive, the evidence that women can and do bias the outcome of sperm competition by orgasmic timing is still very weak. At best, all that Baker and Bellis have shown is that women retain more sperm in their reproductive tracts if they experience orgasm between about 1 minute before and 45 minutes after their partner ejaculates than if they have an orgasm earlier or not at all. Even this ostensible fact requires not just replication but confirmation using other techniques, for as it stands, the inference is premised on the assumption that the number of sperm ejaculated is identical regardless of whether or when female orgasm occurs, so that sperm retention can be computed from an estimate of that ejaculate minus "flowback." This may not be true, since the duration of pre-ejaculatory male sexual arousal is most likely confounded with the criterion used to categorise female orgasms as either low or high sperm retention (i.e., whether they occur before or after male ejaculation). This is important since the number of sperm ejaculated by human males is positively correlated with the duration of pre-ejaculatory sexual arousal for specimens collected during copulation (Zavos 1988) and via masturbation (Pound et al. 1999). Consequently, Baker and Bellis's estimates of the number of sperm retained for copulatory episodes during which the female has an orgasm after the male are likely to be based on overestimates of the number of sperm ejaculated. The "high sperm retention" (HSR) orgasm may thus be simply an artifact of a method that fails to take into account an important determinant of ejaculate composition.

In any event, even if female orgasms can affect the number of sperm retained, the impact of biased retention on the outcome of sperm competition needs to be assessed; it could be substantial or negligible. At present there is no evidence that female orgasms, copulatory or otherwise, have any effect on conception rates, as one might expect if "HSR" orgasms really improve the retained sperm's chances of encountering the ovum, and more crucially, it remains to be shown that a woman who copulates with two men during a single fertile period is more likely to conceive a child sired by the one with whom she experiences a copulatory orgasm.

This may seem like quibbling, since it is the conceptually integrated story of contingent female orgasmic response that makes the hypothesis of post-copulatory female choice so plausible. But the story of contingent female response is itself still just an hypothesis, too, albeit a strong one. Thornhill et al. (1995) found that women with low-FA partners reported more frequent HSR orgasms (by the above timing criteria) than women with high-FA partners. But although Thornhill et al. found no evidence that women with low-FA partners are simply more orgasmic than other women, their main finding is nevertheless a between-subjects result. They did not establish that individual women vary their orgasmic behavior in relation to partner's FA, and it remains possible that those women who are most likely to have copulatory orgasms are those most likely to have low-FA partners. Moreover, even a demonstration of Thornhill et al.'s effect within individual female respondents would not close the question. The women were merely asked how often they attained orgasm before, after, or at the same time as their partners during sexual intercourse, a question that cannot show that it is the women who are responding differentially to men varying in FA; an alternative hypothesis is that low-FA men ejaculate sooner. Finally, Thornhill et al. (1995) define HSR orgasms as those that occurred during sexual intercourse after (or at the same time as) male ejaculation, but this definition may not be ideal; it excludes, for example, cases in which the penis is withdrawn after ejaculation, but sexual stimulation is continued and the woman reaches orgasm soon after, even though these would seem likely to also be HSR orgasms according to the Baker and Bellis scenario.

There is variability in the frequency with which women have or- 
gasms, in their timing relative to partner orgasm, and in the sexual activities during which they occur, but we do not yet know how much of this variability can be attributed to relatively stable differences among females, and how much to attributes of their partners. These two sources of variability will undoubtedly prove to be confounded, and yet they must be separated before we can be confident that female orgasm really functions to bias the outcome of sperm competition.

\section{Analyses of mating differences within-sex and between-sex are complementary, not competing}

Todd K. Shackelford, Gregory J. LeBlanc, Richard L. Michalski, and Viviana A. Weekes

Division of Science-Psychology, Florida Atlantic University, Davie, FL 33314. \{tshackel; gleb2044; rmic5640; vwee9812\}@fau.edu

\begin{abstract}
Analyses of between-sex differences have provided a powerful starting point for evolutionarily informed work on human sexuality. This early work set the stage for an evolutionary analysis of within-sex differences in human sexuality. A comprehensive theory of human sexual strategies must address both between-sex differences and within-sex differences in evolved psychology and manifest behavior.
\end{abstract}

Gangestad \& Simpson (G\&S) present a clear and convincing theoretical case for the evolution of conditional mating strategies in human males and females. In addition, the authors present an organized and impressive array of empirical data corroborating their theoretical claims. G\&S's analysis of the conditional mating strategies of humans relies on several cornerstones of modern evolutionary psychological science. These cornerstones include an appreciation that adaptations are not optimally designed; that adaptations have both costs and benefits in reproductive currencies; that organisms face "trade-offs" in how a limited quantity of time and energy can be allocated to solving different adaptive problems; and that adaptations do not operate in a vacuum, but instead require input for their operation (Dawkins 1976; Williams 1966).

G\&S have significantly advanced the field of human sexuality by providing a cogent analysis of within-sex differences in male and female sexual strategies. The authors take as a starting point that previous work on human sexuality informed by an evolutionary perspective (e.g., Buss 1989; Buss \& Schmitt 1993) overemphasized between-sex differences. They contend that previous work paints a picture of human males as exclusively interested in short-term sexual relationships and of human females as exclusively interested in long-term, committed relationships. early work in human sexuality informed by an evolutionary psychological perspective addressed sex differences in mating psychology and behavior, in part because these differences are so cleanly and clearly predicted from straightforward evolutionary logic (Symons 1979; Trivers 1972; for review, see Buss 1994b). None of this early work, however, discounted the reality of within-sex differences in sexual behavior and psychology. Instead, much of this early work noted that a weakness of evolutionarily-informed analyses of human sexuality is that within-sex differences were not fully explicable based on then-current theoretical accounts (see, e.g., Buss 1994b; Buss \& Schmitt 1993; DeKay \& Buss 1992).

The early focus on differences between the sexes in sexuality was a reasonable and powerful starting point for evolutionary psychological work on human sexuality. G\&S correctly note that this early work failed to successfully explain within-sex differences in mating. But this was not the intent of this early work on human sexuality informed by an evolutionary perspective. The intent of this early work was to document predictable and on-average differences between men and women in their mating desires and behaviors. This focus on sex differences soon brought to the fore- front the presence of substantial within-sex differences. A comprehensive and empirically supported analysis of within-sex differences in human sexuality awaited the insight of researchers such as G\&S.

G\&S provide a missing piece to the puzzle of human mating psychology and behavior. Men and women, on average, clearly differ in several key features of sexual psychology and behavior. All men are not alike, however, and neither are all women alike. We now have a comprehensive theory and supportive empirical work to explain these within-sex differences. Between-sex differences and within-sex differences are different and complementary, not competing, levels of analysis. One need not argue that there are either between-sex differences or within-sex differences. There are both.

The thrust of the target article is that within-sex differences are substantial and worthy of focused theoretical and empirical work. We agree, and we expect that researchers who have produced the work on sex differences in human sexuality also agree. We are concerned, however, that some readers might misunderstand G\&S as arguing that within-sex differences are more important than between-sex differences in human sexuality. This sort of misunderstanding is akin to arguing that the neurobiological level of analysis is more important than the psychological level of analysis in understanding human cognition. Neither level of analysis is more important; instead, they are equally important but definitively different levels of analysis. And so is it the case with analyses of between-sex differences and within-sex differences in human sexual psychology and behavior. Previous work by researchers such as Buss and Schmitt (1993) advanced the field of between-sex differences. G\&S provide a valuable contribution to the emerging field of within-sex differences. A comprehensive theory of human sexuality must address both levels of analysis.

\section{Sexual attractiveness: Sex differences and overlap in criteria}

\section{John Marshall Townsend}

Department of Anthropology, The Maxwell School, Syracuse University, Syracuse, NY 13244-1090. jmtsu44@aol.com

\begin{abstract}
Women with high sociosexual orientation inventory (SOI) scores may trade signs of willingness to invest for signs of ability to invest, instead of, or in addition to, genetic benefits. The target person's status traits affect women's judgments of sexual/physical attractiveness more than men's. An objective measure of a physical trait such as FA is therefore crucial in untangling the factors affecting women's judgments of attractiveness.
\end{abstract}

Gangestad \& Simpson (G\&S) propose an interesting thesis and provide a thorough analysis of pertinent literature to support it. Their argument has important implications for research on sexual attractiveness and mate selection and deserves to be explored from a variety of angles. I wish to clarify only a few points.

In real life, both sexes' judgments of attractiveness are typically affected by both physical and social traits (Townsend 1989; 1993; 1998). In some conditions, however, one sex may be unaffected by traits that significantly affect the other. Male law students, but not females, were affected by models' physical attractiveness when models were portrayed as having high status (Townsend \& Roberts 1993). Women's judgments, but not men's, of physical, dating, or sexual attractiveness were affected by peer opinion (Graziano et al. 1993), target persons' costume (Townsend \& Levy 1990), dominance (Sadalla et al. 1987), and apparent socioeconomic status (Townsend \& Wasserman 1998). Consequently, when studies indicate that some women (e.g., women with high SOI scores) place more emphasis on physical attractiveness (e.g., Townsend 1993), it is not certain whether the traits assessed as "physical attractiveness" are physical, social, or both. An objective 